\title{
Relationship between blocking temperature and strength of interparticle interaction in magnetic nanoparticle systems
}

\author{
M. S. Seehra* and K. L. Pisane
}

Department of Physics \& Astronomy, West Virginia University, Morgantown, WV 26506, USA

\begin{abstract}
In magnetic nanoparticle systems, the variation of the blocking temperature $T_{B}$ with the measuring frequency $f_{m}$ is often used to determine the strength of the interparticle interactions (IPI) through a parameter $\Phi$ or the Vogel-Fulcher temperature $T_{o}$. Presence of IPI is inferred if $T_{o}>0$ and $\Phi=\Delta T_{B} /\left[T_{B} \Delta \log _{10} f_{m}\right]<0.13$ where $\Delta$ signifies changes in $T_{B}$ and $f_{m}$. Here it is shown that these two parameters are related by the Eq. $\Phi=\Phi_{o}\left[1-\left(T_{o} / T_{B}(1)\right)\right]$ where $\Phi_{o} \approx 0.11$ to 0.15 is a constant of the system depending on the magnitudes of measuring frequency and the attempt frequency $f_{o}$ of the Néel relaxation. Using data on a variety of nanoparticle systems, experimental verification of this relationship is also presented.
\end{abstract}

*Corresponding author. Email: mseehra@wvu.edu; Phone: 304-293-5098

Key words: Magnetic nanoparticles; interparticle interactions; blocking temperatures; VogelFulcher relation. 


\section{Introduction}

Magnetic properties of materials in reduced dimensions (thin films, wires and nanoparticles) continue to attract world-wide attention for two reasons: the emergence of new scientific phenomenon and potential applications of materials with reduced dimensions [1-4]. For magnetic nanoparticles (NPs), potential applications include tumor therapy by magnetic hyperthermia, targeted dug delivery, MRI contrast agents and biosensors [5-10]. An important property of magnetic NPs is their blocking temperature $T_{B}$ which separates the superparamagnetic (SPM) state for temperatures $T>T_{B}$ from the frozen magnetic state for $T<T_{B}$. For many biomedical applications, the NPs need to be in the SPM state at room temperature requiring $T_{B}<300 \mathrm{~K}$ so that the NPs are only magnetic in an applied magnetic field $H$ without any residual magnetization $(M)$ when $H$ is removed. Thus determination of the blocking temperature of a nanoparticle system is usually the first property to be measured.

For defining the blocking temperature $T_{B}$, consider a system of nanoparticles (NPs) each with volume $V$ and uniaxial anisotropy constant $K_{a}$. At a temperature $T$ and in the absence of any interparticle interactions (IPI), the rate of flipping $f$ of the magnetic moment of the particle against the energy barrier $E_{a}=K_{a} V$ is given by the Néel-Brown relaxation [11, 12]:

$$
f=f_{o} \exp \left(-E_{a} / k_{B} T\right) \text {-------- (1). }
$$

In Eq. (1), $k_{B}$ is the Boltzmann constant and $f_{o}$ is the system-dependent attempt frequency varying only weakly with temperature as discussed at some length later. An anisotropy related temperature $T_{a}=E_{a} / k_{B}$ may be conveniently defined. From Eq. (1), when $f$ becomes equal to $f_{m}$, the frequency of measurement, then the system will appear to be blocked below the blocking temperature $T_{B}$ given by 


$$
T_{B}=T_{a} / \ln \left(f_{o} / f_{m}\right)
$$

Eq. (2) is not valid in the presence of IPI whose strength depends on particle concentration, their size and size distributions, and on the nature of the matrix. Contributions to the IPI can come from several sources. For NPs with magnetic moment $\mu$ separated by distance $r, E \sim \mu^{2} / 4 \pi r^{3}$ is the interaction energy from the dipole-dipole interaction. If the particles are uncoated and in close contact, even the exchange interaction may become significant. When the matrix and the particles are both metallic, then the RKKY (Ruderman- Kittel-Kasua-Yosida) interaction varying as $1 / r^{3}$ may become important. In addition, the presence of strong IPI may lead to a low-temperature collective state with long-range order such as super-ferromagnetism or super-spin glass state [13]. Thus, determining the strength of IPI and its effects on the measured properties in a nanoparticle system is an important issue for understanding its magnetic properties.

Computationally [14-16] and experimentally [17-19], it is now known that the presence of IPI raises the magnitude of the blocking temperature $T_{B}$ by an effective temperature $T_{o}$ representing the strength of the IPI among the NPs. For the case of $T_{o}<T_{B}$ Eq. (1) is often replaced by the Vogel-Fulcher law [20-21] given by

$$
f=f_{o} \exp \left[-E_{a} / k_{B}\left(T-T_{o}\right)\right]
$$

Eq. (3) was also proposed by Tholence [21] for spin glasses with the frequency dependence of the freezing temperature $T_{f}$ replacing $T_{B}$. Additional discussion and analysis on the use and limits of Eq. (3) for super-paramagnets and spin glasses is given in the paper by Dormann et al [22]. The use of Eq. (3) leads to a new relationship for $T_{B}$, replacing Eq. (2), in which $T_{B}$ is enhanced by $T_{o}$ :

$$
T_{B}=T_{o}+T_{a} / \ln \left(f_{o} / f_{m}\right) \text {------- (4). }
$$


Experimentally, the presence of IPI in a magnetic nanoparticle system can be detected by comparing the magnitudes of $T_{B}$ at a fixed $f_{m}$ with and without magnetic dilution since $T_{B}$ will be lower with magnetic dilution, such as coating of the particles with a surfactant $[18,19]$. An alternative approach to detecting the presence of IPI is determining how $T_{B}$ in a system varies with the change in the measuring frequency $f_{m}$. For this purpose, a quantity $\Phi$ has been defined as [22]:

$$
\Phi=\Delta T_{B} /\left[T_{B} \Delta \log _{10} f_{m}\right]----------~(5) .
$$

In Eq. $5, \Delta T_{B}$ is the change in $T_{B}$ with change in $f_{m}$ written in terms of $\Delta \log _{10} f_{m} . T_{B}$ is usually defined as the position of the peak in the out-of-phase ac magnetic susceptibility $\chi^{\prime \prime}$ measured at a particular $f_{m}$. The magnitude of $\Phi$ is known to vary with the strength of IPI; $\Phi \geq 0.13$ for noninteracting particles, $0.05<\Phi<0.13$ for interacting particles with $\Phi$ decreasing with increase in the strength of IPI and $\Phi<0.05$ for spin-glasses [22]. Since both $T_{o}$ and $\Phi$ have been used in the literature to provide a measure of the strength of the IPI in a system, it is instructive to determine whether a relationship exists between these two parameters. In this paper, we derive such a relationship and verify its validity using our own yet unpublished data on four oleic-acid coated maghemite NPs with average sizes of $2.5 \mathrm{~nm}, 3.4 \mathrm{~nm}, 6.3 \mathrm{~nm}$ and $7.0 \mathrm{~nm}$ [23] as well as data from published papers in a number of nanoparticle systems where the magnitude of both $T_{o}$ and $\Phi$ are available.

\section{Derivation of the relationship between $T_{o}$ and $\Phi$}

Consider measurements of $T_{B}$ in a system using two different measuring frequencies $f_{m}(1)$ and $f_{m}(2)$ with $f_{m}(2)>f_{m}(1)$. Applying Eq. (4) yields

$$
T_{B}(1)=T_{o}+T_{a} /\left[\ln f_{o}-\ln f_{m}(1)\right]------(6)
$$


and

$$
T_{B}(2)=T_{o}+T_{a} /\left[\ln f_{o}-\ln f_{m}(2)\right]------(7)
$$

Using equations (6) and (7), $\Delta T_{B}=T_{B}(2)-T_{B}(1)$ is calculated and substituted in Eq. (5) to determine $\Phi$. After some simplifications, the following equations are derived without any approximation:

$$
\begin{gathered}
\Phi=\Phi_{o}\left\{1-\left[T_{o} / T_{B}(1)\right]\right\}----(8) \\
\Phi_{o}=2.3026 /\left\{\ln \left[f_{o} / f_{m}(2)\right]\right\} \text {-------- (9). }
\end{gathered}
$$

From Eq. (9), the magnitude of $\Phi_{o}$ is a constant of the system in that it depends on the attempt frequency $f_{o}$ and measuring frequency $f_{m}(2)$. Usually, $f_{o}$ varies between $10^{9} \mathrm{~Hz}$ to $10^{12} \mathrm{~Hz}$ for different systems as discussed later and $f_{m}$ in commercially available experimental systems can be varied from a low value of $0.1 \mathrm{~Hz}$ to high value of $10^{4} \mathrm{~Hz}$. As examples, using Eq. (9), if $f_{o}=10^{10}$ $\left(10^{9}\right) \mathrm{Hz}$ and $f_{m}(2)=10^{3} \mathrm{~Hz}$, then $\Phi_{o}=0.143(0.167)$ is obtained. Similarly for $f_{o}=10^{12} \mathrm{~Hz}$ and $f_{m}(2)=10^{4} \mathrm{~Hz}, \Phi_{o}=0.125$ is obtained. For the often quoted $\Phi_{o}=0.13, f_{o} / f_{m}(2)=4.9 \times 10^{7}$ is needed. Eq. (8) relating $\Phi$ to the fractional change $T_{o} / T_{B}(1)$, along with Eq. (9) for $\Phi_{o}$, and the experimental verification of Eq. (8) given below are the new results of this paper.

\section{Experimental Results and Discussion}

For experimental verification of Eq. (8) connecting $\Phi, T_{o}$ and $\Phi_{o}$, the literature was searched to find magnetic nanoparticle systems where magnitudes of $\Phi, T_{B}(1)$ and $T_{o}$ have been published by the authors. The magnitude of $\Phi$ is easily determined using Eq. (5) if $T_{B}$ is measured for at least two sufficiently different frequencies. Following Eq. (4), determination of $f_{o}$ and $T_{o}$ and consequently $T_{a}$ can be done by plotting $\ln f_{m}$ vs. $1 /\left(T_{B}-T_{o}\right)$ for different choices of $T_{o}$ until 
the data of $T_{B}$ at different $f_{m}$ values falls on a straight line for a given choice of $T_{o}$ [24-27]. The intercept on the ordinate then yields $f_{o}$ and the slope $T_{a}$. Based on these considerations, the plot of $\Phi$ versus $T_{o} / T_{B}(1)$ is shown in Fig. 1 with each data point belonging to a different system. Included here are our data of $\Phi, T_{o}$ and $\Phi_{o}$ on four oleic-acid coated maghemite NPs with average sizes of $2.5 \mathrm{~nm}, 3.4 \mathrm{~nm}, 6.3 \mathrm{~nm}$ and $7.0 \mathrm{~nm}[23,24]$ along with the data on $3 \mathrm{~nm} \mathrm{Fe} / \mathrm{Pt}$ core-shell

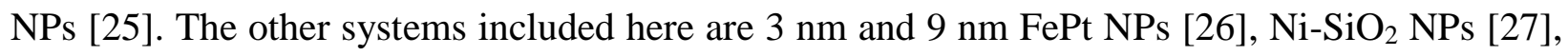
and NiO NPs [28]. The data on the systems $\mathrm{Fe}_{-} \mathrm{Al}_{2} \mathrm{O}_{3}, \mathrm{ZnCr}_{1.6} \mathrm{Ga}_{0.4} \mathrm{O}_{4}$ [29], $\mathrm{CdCr}_{1.7} \mathrm{In}_{0.3} \mathrm{~S}_{4}[30]$ and $\mathrm{CsNiFeF}_{6}[31]$ are tabulated in the paper by Dormann et al [22]. The plot of $\Phi$ versus $T_{o} / T_{B}(1)$ should yield a straight line if Eq. (8) is valid with the intercept yielding the magnitude of $\Phi_{o}$. Overall, the data follows the predicted linear variation of $\Phi$ with $T_{o} / T_{B}(1)$ with the two dotted lines drawn for $\Phi_{o}=0.11$ and $\Phi_{o}=0.15$ bracketing most of the data points. In Fig. 1, the numbers in [ ] adjacent to each system represent reference to the publication from which the data is taken. The scatter in the data points around the predicted linear behavior likely results from different magnitudes of the ratio $f_{o} / f_{m}(2)$ and hence $\Phi_{o}$ in different systems since $f_{o}$ is indeed somewhat system dependent and not a constant as discussed below.

The Brown model for thermal relaxation of superparamagnetic NPs [12, 22] gives the following equation for $f_{o}$ :

$$
f_{o}=2 \gamma_{o}\left(T_{a} / \pi T\right)^{1 / 2} k_{B} T_{a} / \mu_{p}
$$

In Eq. (10), $\gamma_{o}=2 \pi g \mu_{B} / h=1.759 \times 10^{7}$ is the gyromagnetic ratio for $g=2, \mu_{p}$ is the magnetic moment of the NP and $T_{a}$ is the effective anisotropy temperature defined in Eq. (2). According to Eq. (10), $f_{o}$ depends on the system parameters $T_{a}$ and $\mu_{p}$. However, the $T^{-1 / 2}$ temperature dependence of $f_{o}$ evident in Eq. (10) is much weaker than the exponential temperature dependence 
of $f$ in Eq. (2) and Eq. (3) so that $f_{o}$ may be considered to be relatively constant in a limited temperature range where Eq. (2) and Eq. (3) are applied.

As examples of the calculations of $f_{o}$ and $\Phi_{o}$, we use the published parameters for $7.0 \mathrm{~nm}$ NPs of maghemite for which $T_{a}=725 \mathrm{~K}$ and $\mu_{p}=7500 \mu_{B}$ have been reported [24]. Substituting these values in Eq. (10) yields $f_{o}=44 \mathrm{GHz}$ at $T=300 \mathrm{~K}$, comparing favorably with the experimental $f_{o}=26 \mathrm{GHz}$ determined from fitting the data of $T_{B}$ at different $f_{m}$ to Eq. (4). If $T=$ $T_{B}=35 \mathrm{~K}$ is used, then $f_{o}=129 \mathrm{GHz}$ is obtained. Using $f_{o}=26 \mathrm{GHz}(129 \mathrm{GHz})$ and experimental $f_{m}(2)=5 \mathrm{kHz}$ in Eq. (9) yields $\Phi_{o}=0.149(0.135)$. This illustrates some system and temperature dependent variations in the magnitude of $\Phi_{o}$ as argued above.

\section{Conclusions}

In this paper, it is shown that the two parameters $\Phi$ and $T_{o}$ usually used to determine the strength of the interparticle interactions in magnetic nanoparticle systems are related by Eq. (8) with $\Phi_{o}$ given by Eq. (9). The experimental verification of this relation is presented in Fig. 1 using data on a variety of nanoparticle systems. The magnitude of $\Phi_{o} \approx 0.13$ often quoted in papers really depends on the ratio $f_{o} / f_{m}(2)$ for each system and so it may differ somewhat from this magnitude as shown in representative calculations given above. The above derivation depends on the validity of the Vogel-Fulcher relation (Eq. 3) for a system.

\section{Acknowledgement}

This work was supported in part by a grant from the U.S. National Science Foundation, Grant \# DGE-1144676. 


\section{Figure Captions:}

Figure 1: Verification of Eq. (8) by plotting $\Phi$ versus $\left(T_{o} / T_{B}\right)$ for different systems listed in the legend of the figure. The solid circles are experimental data points with experimental uncertainties shown for cases where this information was available. The two dotted lines represent Eq. (8) with $\Phi_{o}=0.15$ and 0.11 . The numbers in [ ] give reference to the paper from which data are taken. 


\section{References}

1. D. Fiorani (Ed.) Surface Effects in Magnetic Nanoparticles, Springer, New York, NY 2005

2. S.P. Gubin (Ed.) Magnetic Nanoparticles, Wiley-WCH, Weinheim, Germany, 2008.

3. N.T.K. Thanh (Ed.). Magnetic Nanoparticles: From Fabrication to Clinical Applications, CRC Press, Boca Raton, FL. 2012

4. D.J. Sellmyer, M. Zheng, and R. Skomski, J. Phys.: Condens. Matter 13, (2001) R433.

5. Q.A. Pankhurst, N.T.K. Thanh, S.K. Jones, J. Dobson, J. Phys. D: Appl. Phys. $\underline{42}$, (2009) 224001.

6. C.C. Berry, A.S.G. Curtis, J. Phys. D: Appl. Phys. 36 (2003) R198

7. C.. Wang, S. Ravi, G.V. Martinez, V. Chinnasamy, P. Raulji, M. Howell, Y. Davis, J. Mallela, M.S. Seehra, S. Mohapatra, J. Control. Release. 136 (2012) 82.

8. E. Rosensweig, J. Magn. Magn. Mater. 252 (2002) 370

9. R. Hergt, S Dutz, J. Magn. Magn. Mater. 311 (2007) 187.

10. S. Maenosono, T. Suzuki, S. Saito, J. Magn. Magn. Mater. 320 (2008) L79.

11. L. Néel, Ann. Geophys. 5 (1949) 99.

12. W. F. Brown, Phys. Rev. 130 (1963) 1677.

13. B. Bedanta and W. Kleeman, J. Phys. D.:Appl. Phys.42 (2009)013001.

14. R.W.Chantrell, N. Walmsley, J. Gore, M. Maylin, Phys. Rev. B 63 (2000) 024410

15. D. Kechrakos, K. N. Trohidu, Phys. Rev. B 58 (1998) 12169.

16. P. Allia et al. Phys. Rev. B 64 (2001) 144420

17. F. Bodker, M.F. Hansen, C. Bender Koch, S. Morup, J. Magn. Magn. Mater. 221(2000) 32.

18. H. Shim, P. Dutta, M.S. Seehra, J. Bonevich, Solid St. Commun. 145, (2008) 192.

19. M. Knobel, W. C. Nunes, H. Winnischofer, T.C.R. Rocha, L.M. Socolovsky, C. L. Mayorga, 
D. Zanchet, J. Non-Cryst. Solids 153 (2007) 743.

20. S. Shtrikman, E. P. Wohlfarth, Phys. Lett. 85A (1981) 467.

21. J. L. Tholence, Solid State Commun. 35 (1980)113

22. J.L. Dormann, L. Bessais, D. Fiorani, J. Phys. C: Solid St. Phys. 21 (1988) 2015.

23. Ph.D. dissertation of K. L. Pisane entitled "Effects of Size and Size Distribution on the Magnetic Properties of Maghemite Nanoparticles and Iron-Platinum Core-Shell Nanoparticles” West Virginia University, Morgantown, WV, USA (2015, unpublished).

24. K. Pisane, E. Despeaux, M.S. Seehra, J. Magn. Magn. Mater. 384 (2015) 148.

25. K. Pisane, S. Singh, M.S. Seehra, J. Appl. Phys. 117 (2015) 17D708.

26. M.S. Seehra, V. Singh, P. Dutta, S. Neeleshwar, Y.Y. Chen, C.L. Chen, S.W. Chou, C.C. Chen, J. Phys. D: Appl. Phys. 43 (2010) 145002.

27. V. Singh, M. S. Seehra, J. Bonevich, J. Appl. Phys. 105 (2009) $07 \mathrm{~B} 518$.

28. H. Shim, A. Manivannan, M.S. Seehra, K.M. Reddy, A. Punnoose, J. Appl. Phys. 99 (2006) 08Q503.

29. D. Fiorani, S. Viticoli, J.L. Dormann, J.L. Tholence, A.P. Murani, Phys. Rev. B 30 (1984) 2776.

30. E. Vincent, J. Hammann and M. Alba, Solid St. Commun. 58 (1986) 57.

31.C. Pappa, J. Hammann, G. Jehanno, C. Jacoboni, J. Phys. C: Solid St. Phys. 18 (1985) 2817. 


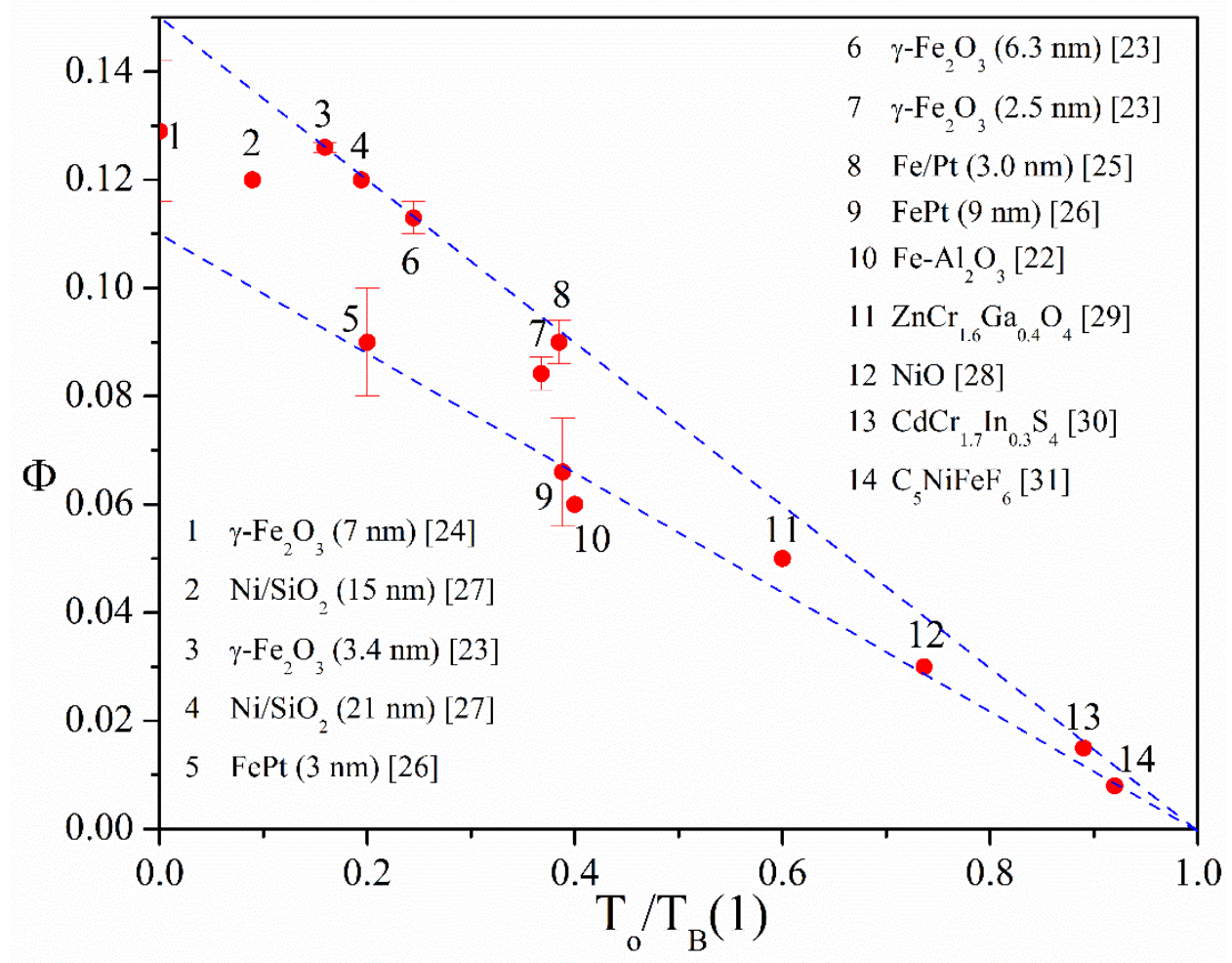

Figure 1: Verification of Eq. (8) by plotting $\Phi$ versus $\left(T_{o} / T_{B}(1)\right)$ for different systems listed in the legend of the figure. The solid circles are experimental data points with experimental uncertainties shown for cases where this information was available. The two dotted lines represent Eq. (8) with $\Phi_{o}=0.15$ and 0.11 . The numbers in [ ] give reference to the paper from which data are taken. 